\title{
NI LAS PERSONAS SON UNA MÁQUINA, NI LA SALUD UNA MERCANCÍA RIESGOS PSICOSOCIALES EN EL TRABAJO MINERO METALÍFERO
}

\author{
Margarita Moscheni y Valeria Gili Diez
}

\begin{abstract}
Resumen
El trabajo puede llegar a ser fuente de satisfacciones y también de sufrimientos, dependiendo del contenido y la organización del modo de producción imperante. Sus características protegen o exponen a ciertos riesgos psicosociales en el ámbito laboral y es propósito de este artículo identificar dichos riesgos en la minería metalífera. Para ello, desde una mirada interpretativa, particularmente cualitativa, se toma como estudio de caso a los trabajadores de San Juan, Argentina, en 2019. Como resultado, se detectaron distintos factores que causan riesgos y sus consecuencias sociales y en la salud física y psíquica. Entre ellos, uno de los más importantes es el de la ruptura de los vínculos afectivos de los trabajadores, por ejemplo con sus familias.
\end{abstract}

Palabras clave: riesgos psicosociales, trabajo, minería.

\begin{abstract}
Neither is the man a machine nor is health a commodity. Psychosocial risks of working in the metalliferous mining sector

Work can be a source of satisfaction, but also a cause of suffering. According to some theorist's expert on the topic, this depends on the content and the organization of the dominant production method. Its characteristics provide either protection or exposure to psychosocial risks in the workplace, thus it is the purpose of this paper to identify such risks in the metalliferous mining sector. To that end, and from an interpretative viewpoint, specifically a qualitative one, we took as a case study people working in San Juan, Argentina, in the year 2019. The study detected various factors causing risks and their social consequences, as well as their effects on physical and mental health, the most important being the breaking of employees' emotional bonds, such as family ones.
\end{abstract}

Keywords: psychosocial risks, work, mining.

Margarita Moscheni: Licenciada y profesora en Ciencias Políticas. Magíster en Desarrollo Económico. Doctora en Ciencias Sociales. Docente titular de Teoría y Técnica de las Políticas Públicas. Instituto de Investigaciones Socioeconómicas, Facultad de Ciencias Sociales, Universidad Nacional de San Juan. CONICET, Argentina.

ORCID iD: 0000-0001-5617-9183

Email: margarita.moscheni@gmail.com

Valeria Gili Diez: Licenciada y profesora en Sociología. Doctora en Estudios Sociales Agrarios. Docente titular de la Facultad de Ciencias Sociales, Universidad Nacional de San Juan. Argentina.

ORCID iD: 0000-0003-1662-995?

Email:valeriagili@unsj-cuim.edu.ar 


\section{Introducción}

El presente artículo tiene como objeto de análisis los riesgos psicosociales en el trabajo (en adelante, RPST) en la minería metalífera de la provincia de San Juan, Argentina, profundizando en la dimensión subjetiva del trabajo.

Partimos de considerar al trabajo como una actividad que no es perjudicial en sí misma, sino que es el contenido y la organización del modo de producción imperante, en ocasiones deshumanizador, lo que constituye la principal causa de los RPST. En este sentido, analizamos la relación que se establece entre trabajo, salud y enfermedad en la minería metalífera en el marco del modo de producción vigente.

A partir de 2003, el advenimiento de la minería metalífera en San Juan, con su lógica trasnacional, constituyó un hito transformador de la organización del trabajo en la provincia. Se radicaron distintos megaproyectos mineros: Veladero (2003), Gualcamayo (2007), Casposo (2009) y Lama (2009), que instalaron a la minería como actividad hegemónica local. A nivel de la superestructura, se instaló un discurso que pregonaba las múltiples virtudes del trabajo minero, destacándose entre ellas el alto nivel de remuneración con relación a las demás actividades que conformaban el perfil productivo provincial.

Los RPST se observan tanto en la salud física como en la psíquica de los trabajadores. Sin embargo, las estadísticas dan cuenta solo de los casos de accidentes laborales que impactan en la dimensión física, relegando e invisibilizando las consecuencias psicosociales, que son objeto de este artículo.

Metodológicamente, el trabajo se abordó desde el enfoque cualitativo, con la estrategia estudio de caso, atendiendo a los riesgos en los trabajadores de la minería metalífera en San Juan. Se considera que el abordaje cualitativo es el más adecuado, en tanto permite comprender e interpretar posibles narrativas (dentro de todo un universo), no como informaciones ni como verdades, sino como un discurso que es interpretado en su habla y en su silencio por un determinado sujeto histórico (De Souza, 2010): el trabajador minero en San Juan, en este trabajo. En cuanto a la elección de la estrategia — estudio de caso- responde a la necesidad de profundizar en las particularidades, delimitando en tiempo y espacio (Neiman y Quaranta, 2013) y a que, a diferencia de otras estrategias en investigación cualitativa, existe un marco conceptual como punto de partida — el de los RPST — , que recorta el estudio a la realidad social circundante del objeto. 
La principal técnica de construcción de datos fue la entrevista semiestructurada y las unidades de análisis fueron los trabajadores mineros en San Juan. La muestra fue no probabilística de tipo intencional o dirigida, seleccionando las unidades a partir de distintas variables que consideramos pueden influir en la generación de ciertos RPST, tales como el género, la edad, la antigüedad, el proyecto minero, el puesto ocupado y si es jerárquico o no.

De esta manera, en enero de 2019 se realizaron catorce entrevistas semiestructuradas a trabajadores mineros, distribuidos como lo muestra el Cuadro 1.

Cuadro 1. Caracterización-perfil de las unidades de análisis

\begin{tabular}{|c|c|c|c|c|c|c|}
\hline N. ${ }^{\circ}$ caso & Género & Edad & Antigüedad & Mina & Puesto & Jerárquico \\
\hline 1 & $\mathrm{~F}$ & 30 & 3 años & $\begin{array}{l}\text { Proyecto } \\
\text { Casposo }\end{array}$ & Supervisora & Sí \\
\hline 2 & M & 36 & 7 años & $\begin{array}{c}\text { Proyecto } \\
\text { Lama }\end{array}$ & $\begin{array}{l}\text { Higiene, Seguridad y } \\
\text { Medio Ambiente }\end{array}$ & Sí \\
\hline 3 & M & 55 & 16 años & $\begin{array}{c}\text { Proyecto } \\
\text { Lama }\end{array}$ & Supervisor & Sí \\
\hline 4 & M & 40 & 5 años & $\begin{array}{l}\text { Proyecto } \\
\text { Veladero }\end{array}$ & Almacén & No \\
\hline 5 & M & 49 & 8 años & $\begin{array}{l}\text { Proyecto } \\
\text { Veladero }\end{array}$ & Encargado & No \\
\hline 6 & M & 37 & 5 años & $\begin{array}{l}\text { Proyecto } \\
\text { Veladero }\end{array}$ & Operador de grúa & No \\
\hline$?$ & M & 34 & 4 años & $\begin{array}{l}\text { Proyecto } \\
\text { Veladero }\end{array}$ & $\begin{array}{c}\text { Operador de camión } \\
\text { fuera de ruta }\end{array}$ & No \\
\hline 8 & M & 43 & 13 años & $\begin{array}{l}\text { Proyecto } \\
\text { Veladero } \\
\end{array}$ & $\begin{array}{l}\text { Técnico de control en } \\
\text { mina }\end{array}$ & No \\
\hline 9 & M & 34 & 10 años & $\begin{array}{l}\text { Proyecto } \\
\text { Veladero }\end{array}$ & Mecánico & No \\
\hline 10 & M & 28 & 2 años & $\begin{array}{l}\text { Proyecto } \\
\text { Gualcamayo }\end{array}$ & Personal de seguridad & No \\
\hline 11 & M & 39 & 8 años & $\begin{array}{l}\text { Proyecto } \\
\text { Veladero }\end{array}$ & Médico & Sí \\
\hline 12 & $\mathrm{~F}$ & 36 & $?$ años & $\begin{array}{l}\text { Proyecto } \\
\text { Gualcamayo }\end{array}$ & Administrativa & No \\
\hline 13 & $\mathrm{~F}$ & 33 & 5 años & $\begin{array}{l}\text { Proyecto } \\
\text { Veladero }\end{array}$ & $\begin{array}{c}\text { Operadora } \\
\text { multifunción }\end{array}$ & No \\
\hline 14 & $\mathrm{~F}$ & 42 & 3 años & $\begin{array}{l}\text { Proyecto } \\
\text { Veladero }\end{array}$ & $\begin{array}{l}\text { Higiene, Seguridad y } \\
\text { Medio Ambiente }\end{array}$ & No \\
\hline
\end{tabular}

Fuente: Elaboración propia. 
Los ejes de indagación fueron las relaciones sociales en la actividad laboral y en la familia, la estabilidad en el empleo, la demanda psicológica, el esfuerzo requerido para ejecutar la actividad, la autonomía en las tareas, las exigencias emocionales y los conflictos éticos o de valores.

También se realizaron ocho entrevistas semiestructuradas a secretarios generales de sindicatos vinculados a la trama minera, gerentes del área de una empresa operadora, entre otros informantes claves. El estudio se complementó con datos secundarios, tales como estadísticas, informes e investigaciones especializadas en la temática.

Respecto del análisis, estuvo basado en la tradición comparativa de los estudios de casos, usando la estrategia de comparación analítica, identificando el surgimiento de riesgos psicosociales como producto del trabajo en mina.

Algunos de los hallazgos indican que la dinámica propia del trabajo minero atenta directamente contra la calidad de vida de sus trabajadores. La distancia entre la mina — su lugar de trabajo — y la ciudad — donde residen sus afectos-, y las consecuentes dificultades para mantener vínculos afectivos con sus familias y grupos de pares, sumadas a la exposición a altas temperaturas, el descanso inadecuado, el roster (jornada-rotación laboral), el aislamiento, entre otras dimensiones, generan impactos en la relación salud, trabajo y enfermedad.

\section{Los factores de riesgos psicosociales en el trabajo}

Las condiciones y el ambiente laboral y su vinculación con la salud de los trabajadores conforman un debate de larga data en diversos espacios académicos y de gestión. A comienzos del siglo XX, a impulso de la medicina clásica y la ingeniería, se comenzó a analizar la relación entre salud y trabajo, poniendo el foco en accidentes y enfermedades que afectaban principalmente la dimensión física, desde una visión individualista y en pos de la reparación de daños (Henry, 2019).

Desde el paradigma etiopatogénico se buscaba definir los factores de riesgo presentes en el medioambiente de trabajo indicando relaciones de causalidad directas. Sin embargo, este paradigma generó resistencias y autores italianos como Bonino (1979) y Basaglia (1978) pusieron en discusión el modelo médico hegemónico característico de las sociedades capitalistas. Desde esta línea de pensamiento, se propuso considerar la práctica médica como procesos más estructurales que explican la salud-enfermedad, atendiendo a aspectos sociales, económicos e ideológicos.

En la década del ochenta, en América Latina comenzaron a desarrollarse diversos estudios que muestran un creciente interés por las condiciones de trabajo y la salud de los trabajadores.

En particular, el estudio de los RPST surge a fines del siglo XX, en un contexto de crisis y fuertes procesos de restructuración de la economía mun- 
dial y de introducción de nuevas tecnologías. Los cambios en los procesos de trabajo generan la necesidad de nuevos enfoques para analizar el ambiente laboral. La preocupación creciente por atender a los factores psicosociales se vuelve cada vez más central, sin dejar de lado los efectos tradicionalmente analizados, como los físicos, químicos, biológicos o mecánicos, entre otros.

Por su parte, Michel Gollac, articulando las condiciones y el medioambiente de trabajo (en adelante, CyMAT), define a los RPST como:

los riesgos para la salud, física, psíquica, mental y social engendrados por los determinantes socioeconómicos, la condición de empleo, la organización y el contenido del trabajo y los factores relacionales que intervienen en el funcionamiento psíquico y mental de los trabajadores (Gollac, 2013, citado en Neffa, 2017, p. 71).

De esta manera, observamos que ambas dimensiones - RPST y CyMAT - son indivisibles y afectan la salud (física y psíquica) de los asalariados, que están dotados de un conjunto de saberes prácticos y habilidades cognitivas que les permiten vivenciar los efectos que las condiciones de trabajo poseen sobre sus propias vidas.

Los efectos de los RPST sobre la salud de los trabajadores no son homogéneos, sino que están atravesados por el género, la edad, los sectores económicos y ramas de actividad, las dimensiones de la unidad productiva, el sector (público o privado) y el tipo de puesto ocupado, entre otros. Sin embargo, en la bibliografía consultada observamos relativa unanimidad acerca de la escasa información y toma de conciencia de los trabajadores acerca de la importancia de los RPST y su impacto sobre la salud.

Neffa explica que existen determinantes estructurales de los RPST, que cumplen el papel de variables independientes, como lo son "las condiciones de empleo, la organización y el contenido del proceso de trabajo y las relaciones sociales en el trabajo que son susceptibles de interactuar con impactos sobre la salud psíquica, mental, social e incluso física de los trabajadores" (Neffa, 2017, p. 72).

Estas causas generan gran fatiga y estrés, que predisponen e incluso provocan accidentes de trabajo y hasta la muerte de los trabajadores. Si bien no existe consenso acerca del concepto de RPST ni las formas de medirlo, sí hay acuerdo respecto del papel fundamental que poseen sobre:

las exigencias del contenido y la organización del trabajo, los modos de gestión de la fuerza de trabajo y de las relaciones laborales, y también el impacto de los valores y expectativas de los asalariados (desarrollo de sus competencias, posibilidades de promoción y ascensos, mantener equilibrio entre vida de trabajo y vida familiar y social, evitar los conflictos éticos) (Neffa, 2015, p. 115). 
También se denotan acuerdos acerca de la naturalización que los trabajadores realizan sobre los daños progresivos que provocan los RPST, que llegan a ser percibidos, en última instancia, como ineludibles o como parte de las "reglas del juego". Así, los trabajadores se ven expuestos a resistir o adaptarse, construyendo estrategias de defensa hasta recurrir a

tratamientos de médicos, psicólogos o psiquiatras y a consumir medicamentos, sin comprender que la causa principal es el contenido y la organización del proceso de trabajo. El proceso de trabajo genera determinantes del medio ambiente, emocionales y cognitivos, que constituyen demandas para ejecutar el trabajo prescripto [...] (Neffa, 2017, p. 76).

En estos casos, el sufrimiento - que es patológico- se convierte en normalidad, enfermando por la propia negación, ocultamiento o subestimación de los propios trabajadores con respecto a los efectos de los RPST en su salud.

Es necesario destacar que lo preocupante de la negación o invisibilización para paliar el sufrimiento por parte de los trabajadores es la implicancia de efectos perjudiciales sobre el equilibrio psíquico y mental, que luego se somatizan. Laurell (1993) plantea que la carga laboral es el conjunto de elementos presentes en el proceso de trabajo, que interactúan entre sí y generan desgaste en los trabajadores, lo que implica pérdida de capacidades presentes o potenciales, tanto biológicas como psíquicas (p. 11).

La supremacía otorgada a la dimensión biológica del ser humano es el sustento ontológico sobre el cual se invisibiliza la subjetividad y la propia experiencia de los trabajadores acerca de su cuerpo, otorgándoles una franca primacía a los elementos estructurales del trabajo por encima de los componentes simbólicos, afectivos y psíquicos e incidiendo en la percepción acerca de la salud de los trabajadores.

Cabría preguntarse, entonces, cómo se vincula la salud con el trabajo (Korinfeld y Mendizábal, 2015; Fischer, 2018). Al respecto, Neffa sostiene que esa relación consiste en "lograr la promoción y mantenimiento del más alto grado posible de bienestar físico, mental y social de los trabajadores en todos los trabajos" (2017, p. 96). El autor considera que la salud psíquica y mental no conforma un estado absoluto, sino una homeóstasis permanente entre las necesidades del trabajador y su capacidad de resistencia-adaptación para enfrentar las exigencias físicas, psíquicas y mentales del trabajo.

Atendiendo a lo expuesto hasta aquí, se retoman los aportes de Gollac (2011, 2013, citado en Neffa, 2017, p. 80), quien describe los factores que causan RPST y que pueden adoptar aspectos positivos o negativos para la salud de los trabajadores. Estos se pueden resumir en: la demanda psicológica y el esfuerzo requerido para ejecutar la actividad; las exigencias emocionales; la autonomía en el trabajo; las relaciones sociales en el trabajo (horizontales) 
y las relaciones de trabajo (verticales); y los conflictos éticos o de valores. A continuación se explica brevemente cada uno de ellos.

La demanda psicológica y el esfuerzo requerido para ejecutar la actividad hace referencia a

la cantidad, el ritmo y la intensidad del trabajo que determinan la carga psíquica, mental y global de trabajo, la duración del tiempo de trabajo y su configuración (diurno, nocturno, por turnos, en horarios antisociales), así como la complejidad de la actividad que implica para el trabajador el uso de sus recursos y competencias cognitivas para hacer un trabajo exigente y de calidad asumiendo responsabilidades (Neffa, 2017, p. 80).

Tiempos de trabajo excesivos repercuten negativamente en la salud de los trabajadores, generando perturbaciones en el sueño, del humor y enfermedades cardiovasculares, al tiempo que aumentan las probabilidades de accidentes de trabajo. Especialmente los trabajos nocturnos o por turnos que rotan acrecientan las posibilidades de trastornos de ansiedad, bipolaridad y, especialmente en las mujeres, predisposición al cáncer. Estos tipos de horarios laborales, denominados "horarios antisociales" (Neffa, 2017, p. 80), suelen generar problemas al interior de las familias y los grupos domésticos, al tiempo que existe una mayor propensión a los accidentes de trabajo durante la noche. Dentro de esta dimensión tienen especial importancia las situaciones de trabajadores con una vida física sedentaria y mala alimentación, lo que impacta directamente en su salud.

Dentro de este factor de riesgo se incluyen los problemas de CyMAT que, al no ser contemplados debidamente, generan la necesidad de consumir medicamentos con frecuencia para calmar dolores o diferentes tipos de drogas contra la angustia y la ansiedad.

Lo que se conoce como estrés (daño o tensión física y emocional) habitualmente es considerado como una causa de riesgos psicosociales, sin embargo, Neffa (2017) señala que el estrés en sí mismo no es un trastorno de la salud, sino una consecuencia de un daño emocional o físico, una respuesta a los riesgos psicosociales que incide en el bienestar general de los trabajadores. El estrés se produce cuando hay un desfase entre las exigencias y los recursos que las personas perciben para enfrentarlas.

Entre las consecuencias del estrés, Neffa señala los "trastornos mentales y de la conducta como el desgaste, el burnout (agotamiento), la ansiedad y la depresión. También pueden causar deterioro físico como es el caso de las enfermedades cardiovasculares y los trastornos musculo-esqueléticos" (2017, p. 82).

Las exigencias emocionales son factores a veces inherentes al trabajo, ya que suponen en ciertas oportunidades que los trabajadores soporten silenciosamente la violencia, el acoso, las amenazas y hasta los insultos, ocultan- 
do sus propias emociones para evitar conflictos en su actividad. De manera inversa, cuando los trabajadores desempeñan su tarea de forma automática, sin mentir ni con miedo, el trabajo es fuente de satisfacción y libertad.

\section{La autonomía en el trabajo}

consiste en la posibilidad de ser actor, para controlar y conducir su propia vida profesional y tener la posibilidad de participar en la adopción de decisiones, para lo cual debe usar y desarrollar sus calificaciones y competencias profesionales para realizar la tarea y seguir aprendiendo (Neffa, 2017, p. 83).

La excesiva división técnica y social del trabajo que conlleva a trabajos alienantes, es decir, repetitivos, rutinarios, en donde los asalariados no pueden hacer uso pleno de sus facultades mentales y emocionales, va en detrimento de la autonomía de las personas, quienes terminan por subestimarse y no encontrar estímulo para comprometerse con la tarea que desarrollan.

Las relaciones sociales en el trabajo (horizontales) y las relaciones de trabajo (verticales) son entendidas como "la totalidad de los intercambios dinámicos que los sujetos desarrollan con los diferentes actores de su entorno laboral, pueden constituir un aspecto positivo o un factor negativo de los riesgos psicosociales en el trabajo" (Neffa, 2017, p. 87). Son relaciones amplias porque refieren tanto a los compañeros de trabajo como a los jefes directos y la jerarquía, a la organización en sí misma como empleadora y finalmente, cada vez más, a sus familiares, clientes o usuarios.

La importancia de estas relaciones estriba en que a través de ellas los trabajadores llegan a formar parte del equipo y a sentirse reconocidos en lo que a su tarea, competencias y calificaciones refiere. También son importantes estas relaciones ya que conforman el clima social dentro del lugar de trabajo y permiten que este se constituya en un espacio estimulante en el que el trabajador se involucre y no sea víctima de injusticias, discriminación, hostigamiento, entre otras. Asimismo, a través de estas relaciones los trabajadores pueden compartir entre sí sus saberes y habilidades, se sienten útiles y reconocidos.

Ahora bien, en lo que refiere a las relaciones entre los asalariados y la jerarquía, estas pueden ser apropiadas siempre y cuando estén cimentadas en un buen trato y si quienes tienen mayor poder en la estructura jerárquica tienen capacidad de escucha. De manera contraria, la carencia de reconocimiento por parte de las jerarquías puede desmotivar a los trabajadores y ser, además, fuente de afecciones psíquicas y mentales.

Es importante señalar que la justicia, en sus dimensiones organizacional y procedimental, "se refiere a la actividad, la cantidad y el tipo de trabajo que deben asignarse en la organización a todos de manera equitativa según su formación y experiencia profesional"; al tiempo que debe propiciar que los 
trabajadores - en tanto colectivo- " "puedan participar en la adopción de decisiones (con acceso a información, evacuar consultas, formular propuestas) y la posibilidad de cuestionarlas" (Neffa, 2017, p. 89).

Por su parte, los conflictos éticos o de valores

surgen de la multiplicidad de situaciones que los trabajadores vivencian en sus procesos de trabajo y que pueden configurarse como negativas en tanto afectan de modo particular a sus creencias, valores, normas y convicciones, provocando sufrimiento y siendo susceptibles de poder producir daños sobre su salud integral (biopsicosocial) (Neffa, 2017, p. 93).

Este tipo de conflictos surge en situaciones en las que los asalariados no tienen la posibilidad de trabajar en un ambiente de calidad, ya que no se respetan las reglas de la profesión u oficio, o los medios indispensables para el trabajo no funcionan de manera correcta, porque los insumos utilizados son de mala calidad, por problemas de logística general, entre otros. Otro caso típico se refiere a cuando los trabajadores se ven obligados a realizar cosas con las que no están de acuerdo internamente o cuando sienten que su trabajo es inútil, cuando tienen la vivencia de no ser reconocidos moralmente por su labor en la empresa, así como también en situaciones en las que no disponen de autonomía para tomar las decisiones referentes a su tarea o función. En este sentido, Neffa agrega que estos conflictos éticos se suscitan como consecuencia de que la "empresa u organización ponga a los/as trabajadores/as en situación de inferioridad frente a los clientes u obligarlos a prometer cosas que son imposibles" o les exige actuar contra los intereses del cliente con la obligación de no mencionar o esconder información, "la letra chica de los contratos", "ser ambiguo o mentir sobre los problemas de calidad y de funcionamiento del producto" (2017, p. 92). Como consecuencia, cuando los asalariados dejan de lado sus principios éticos, perciben un gran malestar que se expresa en pérdida de autoestima, culpa e incluso descompensaciones psíquicas que suelen conllevar a estrategias defensivas como la hiperactividad o el burnout.

La inseguridad en el empleo

es una situación que puede ocurrir cuando se es víctima del desempleo, pero también cuando este es inminente o constituye una amenaza. Si el trabajo y el empleo se precarizan y el salario es insuficiente, se corre el riesgo de una retrogradación en las categorías del convenio o del estatuto que pueden dejar personal sin tareas luego de fusiones y concentraciones provocadas por procesos de restructuración económica o reformas administrativas (Neffa, 2017, p. 94).

Esta situación se manifiesta típicamente ante la precariedad del trabajo y si los trabajadores no están registrados debidamente en los organismos de seguridad social o también ante situaciones de crisis que pueden conllevar a 
reducción de horas y hasta a despidos masivos. Otra fuente de inseguridad laboral tiene lugar cuando las posibilidades de ascenso se encuentran disminuidas o inhabilitadas o porque existe discriminación o nepotismo. Por otro lado, también se produce cuando las empresas incorporan nuevas tecnologías o formas de organización del trabajo en las que los trabajadores no han sido formados, lo que podría conducir a que no puedan mantenerse en el puesto.

La inseguridad y la inestabilidad laboral constituyen un factor de riesgo para la salud de los trabajadores, "porque eso reduce su grado de control de la situación, por el miedo de perder el empleo, quedarse sin salarios y seguridad social, de ver retrasada su promoción, y tener que reconvertirse para acceder a otro empleo" (Neffa, 2017, p. 94), lo que genera habitualmente ansiedad, culpa o conflictos interpersonales que predisponen a las adicciones como mecanismo compensador. Cuando los trabajadores perciben que la relación salarial puede romperse, se producen diversas patologías físicas y mentales y de agotamiento generalizado para enfrentar los problemas de la vida cotidiana en general.

Asimismo, las reestructuraciones que generan desempleo y cambios de puestos de trabajo propician estrés y la emergencia de perturbaciones psicológicas y físicas. Sumado a ello, los trabajadores continúan con sus rutinas productivas y no demandan más autonomía para evitar las posibilidades de supervisión, al tiempo que aumenta la competitividad entre los compañeros del trabajo con el objeto de preservar el empleo.

Los trabajadores que tienen asegurada la estabilidad laboral se desempeñan en buenas CyMAT, son reconocidos por el valor de su trabajo, poseen perspectivas positivas de hacer carrera y tienen más posibilidades de estar a gusto en su trabajo. Contrariamente, si estos conjuntos de factores de riesgo no son debidamente controlados, conllevan irremediablemente al sufrimiento y provocan daños severos en la salud del colectivo de trabajo.

\section{El trabajo minero en San Juan. Algunos datos de contexto}

La minería metalífera a gran escala no es una actividad que demande de manera directa la mayor cantidad de empleo en la provincia. Según el Observatorio de Empleo y Dinámica Empresarial (2016), en el primer trimestre del 2016 los sectores que más demandaron trabajo en San Juan fueron la industria (17,2\%), el comercio (17\%) y la construcción (13,8\%), que juntos absorbieron el $48 \%$ del trabajo. Para la misma fuente, la minería comprende solo un 3,8\% del empleo local, lo que equivale a menos de un $2 \%$ de la población económicamente activa (PEA) provincial.

Sin embargo, la demanda de trabajo minero requiere de un perfil particular. En general, los trabajadores pertenecen a un grupo etario relativamente joven: la edad promedio oscila entre 35 y 50 años. Para ingresar a la mina deben demostrar un buen estado de salud biológica, ya que la intensidad de la 
jornada y las condiciones geomorfológicas del espacio que implica el trabajo de mina (altura, clima, oxígeno, etcétera) así lo exigen.

La empresa se encarga de hacer rigurosos controles para verificarlo:

Para estar arriba tenés que estar sano, porque tu corazón trabaja más, te sube la presión. Dormís mal, comés mal, estás estresado. Todos esos son factores de riesgo para el corazón. Entonces si sos más propenso... antes de subir arriba... No puede subir cualquiera arriba. Estamos a cuatro mil metros de altura, cualquier empleado que suba a la mina, o cualquier visita que quiera subir, debe ser estudiado antes. Lo que se llama estudio preocupacional. ¿Vos querés subir? Bueno te vas a tener que sacar sangre, vas a tener que orinar, te van a pedir una radiografía de tórax, te van a hacer un electrocardiograma. Tienen que descartar... que tu corazón funcione bien, que los pulmones también, que no seas asmático, por ejemplo, para que cuando estás a cuatro mil metros y falte el oxígeno, puedas subsistir (profesional sanitarista, comunicación personal, 5 de febrero de 2019).

En cuanto a los controles psíquicos y mentales, se realizan varios test antes del ingreso, pero no se repiten luego de haber firmado el contrato. La atención de la empresa en el plano psíquico es relativamente escasa.

El nivel de sindicalización es alto al interior de una mina, ya que los trabajadores son encuadrados por las empresas al inicio de la relación laboral y los convenios son por empresa, es decir, producto de la negociación entre el gran capital (en general trasnacional) e instituciones sindicales con delegados locales. El gremio principal en la primera etapa de una mina es la Unión Obreros de la Construcción de la República Argentina (UOCRA), y su relevancia es tal mientras dura la construcción del campamento y la mina en sí misma. Cuando se inicia la producción del metal, la Asociación de Obreros Mineros Argentinos (AOMA) adquiere mayor importancia en términos de cantidad de afiliados sindicales. Atendiendo a que las minas están en la zona precordillerana, alejadas de toda zona urbana, los campamentos se constituyen como microciudades, los trabajadores habitan allí durante siete o quince días de corridos, dependiendo del roster propuesto por el empleador. Ello implica que, además de las actividades productivas propias de la cadena minera metalífera, se requiere de otros servicios necesarios para la vida cotidiana, tales como la lavandería de ropa, hotelería, gastronomía, limpieza, seguridad e higiene, transporte de pasajeros, gimnasio, kiosco al interior de la mina, etcétera. En este contexto, intervienen, aunque en una proporción muy pequeña, trabajadores afiliados a otras organizaciones sindicales, tales como la Unión Obreros Metalúrgicos (UOM); la Unión Tranviarios Automotor (UTA); el Sindicato de Choferes de Camiones; la Unión de Trabajadores del Turismo, Hoteleros y Gastronómicos de la República Argentina (UTHGRA); la Unión de Trabajadores de Entidades Deportivas y Civiles (UTEDYC); el 
Centro Empleados de Comercio (CEC), entre otros. Cuando los trabajadores poseen categorías que exceden las establecidas por estos convenios, se llaman trabajadores jerárquicos y se nuclean en dos entidades gremiales: por un lado, en la Asociación Sindical del Personal Jerárquico Minero (ASIJEMIN) y, por otro, en la Asociación de Supervisores de la Industria Metalmecánica de la República Argentina (ASIMRA).

El convenio colectivo de trabajo firmado entre el sindicato más importante, AOMA y Barrick para Veladero, proyecto que por su envergadura posee la mayor cantidad de delegados metalíferos en mina, prevé la categorización que se presenta en el Cuadro 2.

\section{Cuadro 2. Categorías y manejo de equipos para los trabajadores directos de Barrick en Veladero, San Juan, 2010}

\begin{tabular}{|c|c|c|}
\hline & Operaciones & Perforista \\
\hline Categoría 0 & $\begin{array}{l}\text { Recién ingresado a la empresa, sin } \\
\text { conocimiento, práctica e instrucción. }\end{array}$ & \\
\hline Categoría 1 & $\begin{array}{l}\text { Dominar al menos uno de los siguientes } \\
\text { equipos: camión fuera de ruta, } \\
\text { wheeldozer, motoniveladora y topadora. }\end{array}$ & $\begin{array}{l}\text { Opera en forma autónoma un tipo de } \\
\text { perforadora. }\end{array}$ \\
\hline Categoría 2 & $\begin{array}{l}\text { Dominar al menos dos de los siguientes } \\
\text { equipos o más: camión fuera de ruta, } \\
\text { wheeldozer, motoniveladora, topadora, } \\
\text { cargadora auxiliar y excavadora auxiliar. }\end{array}$ & $\begin{array}{l}\text { Opera en forma autónoma dos tipos } \\
\text { de perforadoras. }\end{array}$ \\
\hline Categoría 3 & $\begin{array}{l}\text { Dominar al menos tres de los siguientes } \\
\text { equipos o más: camión fuera de ruta, } \\
\text { wheeldozer, motoniveladora, topadora, } \\
\text { cargadora auxiliar, excavadora auxiliar y } \\
\text { PC } 2000 \text {. }\end{array}$ & $\begin{array}{l}\text { Opera en forma autónoma tres tipos } \\
\text { de perforadoras. }\end{array}$ \\
\hline Categoría 4 & $\begin{array}{l}\text { Dominar al menos tres de los siguientes } \\
\text { equipos o más: camión fuera de ruta, } \\
\text { wheeldozer, motoniveladora, topadora, } \\
\text { cargadora auxiliar, excavadora auxiliar, PC } \\
2000 \text { y necesariamente cargadora o pala. }\end{array}$ & $\begin{array}{l}\text { Opera en forma autónoma cuatro tipos } \\
\text { de perforadoras u opera en forma } \\
\text { autónoma tres tipos de perforadoras } \\
\text { y el camión portabarras o cargador } \\
\text { auxiliar para tapado de pozos. }\end{array}$ \\
\hline Categoría 5 & $\begin{array}{l}\text { Dominar al menos tres de los siguientes } \\
\text { equipos o más: camión fuera de ruta, } \\
\text { wheeldozer, motoniveladora, topadora, } \\
\text { cargadora auxiliar, excavadora auxiliar, PC } \\
2000 \text { y necesariamente cargadora y pala. }\end{array}$ & $\begin{array}{l}\text { Opera en forma autónoma todos los } \\
\text { tipos de perforadoras y el camión } \\
\text { portabarras o cargador auxiliar para } \\
\text { tapado de pozos. Además, debe } \\
\text { realizar funciones de líder nocturno. }\end{array}$ \\
\hline
\end{tabular}

Fuente: Convenio colectivo de trabajo Barrick-AOMA, 2010.

Mientras mayor es la categoría, mayor será la cantidad de máquinas que debe operar el trabajador en forma autónoma. En promedio, más del 90\% 
de los trabajadores son hombres, y las mujeres ocupan mayoritariamente los puestos administrativos (finanzas, recursos humanos, ambiente, etcétera) y de hotelería (gastronomía y limpieza). Las operadoras de camión, paleras o conductoras de perforadoras son relativamente pocas.

Estas categorías corresponden a los trabajadores directos, pero no a los indirectos, grupo en el que se encuentra la mayor cantidad de asalariados. Por cada puesto de trabajo se generan tres indirectos, es decir, el demandado por las empresas contratistas. En agosto de 2018 había 2700 trabajadores directos y 6509 indirectos. De hecho, las operadoras se relacionan de manera directa en mayor medida con empresas que con trabajadores. Para ese mismo año las mineras sumaban un total de 3560 empresas proveedoras (Moscheni et al., 2019). Estas se ocupan de toda la diversidad de actividades que no son directamente mineras. En efecto, la cadena minera implica un conjunto de actividades que van desde limpieza, hotelería, alimentación, higiene, seguridad y ambiente, servicios médicos, seguridad, transporte, construcción y demás servicios que se demandan en una mina, tal como se observa en el Cuadro 3.

\section{Cuadro 3. Bienes y servicios utilizados en la cadena minera metalífera por eslabón}

\begin{tabular}{cc}
\hline Eslabón & Bienes y servicios \\
\hline Primario & $\begin{array}{c}\text { Servicios de laboratorio, geológicos, de energía, recursos humanos, catering, } \\
\text { carpas, alquiler de camionetas, seguros, comunicaciones, seguridad, financieros, } \\
\text { ingeniería metalúrgica, salud ocupacional, contabilidad, inmobiliarios, pasajes, } \\
\text { perforación, voladura, movimiento de suelos, gestión de residuos, etcétera. }\end{array}$ \\
\hline Secundario & $\begin{array}{c}\text { Energía, servicio de recursos humanos, higiene y seguridad, catering, alquiler } \\
\text { de camionetas, seguros, residuos, perforación, voladura, movimiento de suelos, } \\
\text { seguridad, pasajes terrestres y aéreos, servicios bancarios, financieros, } \\
\text { ingeniería, metalúrgica, salud ocupacional, contabilidad, servicios médicos, } \\
\text { inmobiliarios, mecánica e informáticos. }\end{array}$ \\
\hline
\end{tabular}

Fuente: Elaboración propia basada en entrevistas a informantes claves y Minera Argentina Gold S.A., 2002, pp. 257 y 331.

Estas actividades son provistas por empresas de distintas ramas y, aunque todos los trabajadores convivan en una misma mina, cada rama tiene su categorización propia y cada empleado un vínculo laboral distinto, dependiendo del empleador. Esto da lugar a una importante heterogeneidad laboral, entre trabajadores directos e indirectos. Las diferencias abren brechas principalmente en el salario, la cantidad de horas trabajadas, la ropa de trabajo, las condiciones de alojamiento en la mina, la capacitación, la posibilidad de ascenso laboral, la autonomía y la estabilidad. 


\section{Los factores de riesgos psicosociales en la minería de San Juan. El emergente riesgo sociofamiliar y sus consecuencias}

La minería es una actividad peligrosa, tanto en materia laboral como ambiental, y se desarrolla a cielo abierto o en mina subterránea. Sin embargo, muchos la eligen por los altos sueldos y la organización del trabajo, que alterna catorce días de descanso. A largo plazo, este factor será uno de los generadores de mayor riesgo psicosocial, tal como se analizará en este apartado.

En la provincia de San Juan, los RPST de un trabajador minero comienzan al momento de iniciar su viaje a la mina. La mina Veladero se encuentra a 350 kilómetros de San Juan y se llega a ella por caminos sinuosos (que pueden quedar obstaculizados por la nieve en época invernal). El proyecto está situado en la cordillera de los Andes, pegado al límite con Chile y lejos de todo asentamiento humano. Para llegar allí, en condiciones climatológicas normales, el mínimo de recorrido es de ocho horas.

$\mathrm{Al}$ arribar, cada minero descansa para incorporarse en la próxima ronda. La organización del trabajo implica turnos de doce horas, a las que se suman dos horas más para los trabajadores destinados al área de socavón, propia de las minas subterráneas. A eso es necesario agregar las dos horas que implican el recorrido entre las habitaciones y la mina, una para llegar, otra para volver. Así lo afirma una trabajadora: "las doce horas las tenés que cumplir arriba de la máquina y hasta que salís y viene el colectivo a buscarte son catorce horas" (operadora de mina, comunicación personal, 12 de diciembre de 2019). Es decir, la jornada laboral implica una mayor carga horaria con relación a otras actividades económicas, y por más salas de recreación que tengan, la fatiga al término del roster prevalece a las ganas de jugar al pimpón.

Una vez que están allí, la mina se convierte en una especie de isla en el medio de la nada, por lo que los trabajadores no tienen otra cosa que hacer más que trabajar, máxime teniendo en cuenta el régimen de trabajo mencionado. La ubicación, las condiciones de acceso y el aislamiento provocan en los trabajadores trastornos de ansiedad que derivan en estrés emocional. Indicadores como la angustia, la caída del pelo, el sentimiento de soledad y los ataques de pánico son frecuentes. Al respecto, afirma un médico de mina:

yo tengo pacientes que entran y se ponen a llorar, empiezan a llorar. No es todos los días, pero tengo la experiencia de gente con ataques de pánico y "quiero bajar". A nadie se le obliga con una pistola en la cabeza. Por supuesto, solicito el acceso a San Juan, con un diagnóstico de angustia y lo bajan (profesional sanitarista, comunicación personal, 5 de febrero de 2019).

Los días siguen en largas jornadas laborales, que prevén una hora de descanso para comer. En las noches, lejos, en silencio, agotados y extrañando, se hace difícil conciliar el sueño y justo cuando se estaban acostumbran- 
do al ritmo de la primera semana, el turno vuelve a cambiar. De esta manera, si habían comenzado el roster trabajando de día y "durmiendo" en la noche, ahora están obligados a trabajar de noche y dormir con la luz del sol, con un día de adaptación mediante. Claramente, el ritmo del sueño, o lo que los especialistas denominan la alteración del ritmo circadiano, empeora. Así lo revela una trabajadora minera al afirmar que "es muy difícil... cuesta muchísimo dormir en el día. Pero en Veladero llegás tan cansada que te dormís, pero a las doce estás despierta, por eso la semana de noche termino muy filtrada" (operadora, comunicación personal, 12 de febrero de 2019).

Por ello, en ocasiones, la toma de ansiolíticos para revertir este fenómeno se convierte en una solución efectiva: "En Veladero se toma mucha pastilla para dormir, Rivotril. La altura hace que te cueste dormir. Yo duermo cinco horas como mucho. Con el paso de los días venís durmiendo mal, hay otros que directamente no duermen" (operador de camión, comunicación personal, 28 de enero de 2019). La falta de sueño, a su vez, causa otros problemas, tales como la somnolencia, la irritabilidad, la adicción a sustancias para mantenerse despierto y otros trastornos de ansiedad.

A ello se le suma la insuficiencia de oxígeno, como consecuencia de la altura a la que se encuentran algunos proyectos mineros. Veladero está a cuatro mil metros sobre el nivel del mar. Allí, la falta de oxígeno, la mayor exposición al sol y las elevadas temperaturas provocan enfermedades por altura, como edema pulmonar (diferente a un edema agudo de pulmón), hipertensión y el "mal de altura", conocido comúnmente como apunamiento, causante de cefaleas, náuseas y vómitos.

Al respecto, afirma un médico de mina:

Yo he bajado, en casi ocho o nueve años de experiencia, seis pacientes con edema pulmonar de altura, una patología propia de la altura; después de los 2500 metros de altura en algunos pacientes que no hay forma de prever con un estudio, con el correr de las horas, no se adapta y se le empiezan a llenar los pulmones de líquido y el paciente consulta porque tiene tos y está agitado y un color azul (profesional sanitarista, comunicación personal, 5 de febrero de 2019).

Todo ello sucede en un roster o rotación de catorce días. En ese período la autoexigencia en cada trabajador es mayor si se espera cobrar el premio por producción o el premio por asistencia perfecta. Según el convenio colectivo de trabajo entre la AOMA y Troy Resources Argentina LTD (operadora de la mina Gualcamayo en 2010), este consiste en un $10 \%$ más de los establecido en el sueldo básico. Enfermarse o agotarse no es un lujo que pueda permitirse cualquiera. Un día de ausencia significa una pérdida del $25 \%$ del premio, dos días, hasta el $50 \%$ y tres o más faltas implicarán la privación total del premio (artículo 16). 
En este sentido, afirma un delegado gremial respecto de su hijo, que también es trabajador minero:

El que transgrede estas cuestiones las paga carísimo y todos saben que es así, entonces no faltan, por ejemplo, el mío — habla de su hijo — se esguinzó un sábado y se compró una tobillera y se vendó, porque iba a subir así, aunque no podía caminar. Le dije que no, que fuéramos al médico y me respondió: "no, porque me van a descontar la producción y el presentismo", que es un montón de guita, como 17 mil pesos (empleado de Lama, comunicación personal, 16 de enero de 2019).

Como consecuencia del roster, el aislamiento, la intensidad y la cantidad de horas demandadas, el trabajador puede convertirse en un "paciente quemado" y con un bajo nivel de productividad. Un médico de mina describe a personas agotadas física y psíquicamente, que llegan a inventar síntomas o hechos falaces para conseguir un permiso que les permita descansar, bajar antes de lo previsto e incluso, en el caso más extremo, ser despedidas (evitando renunciar para cobrar la indemnización).

Afirma el entrevistado:

La gente que hace catorce por catorce, ${ }^{1}$ que lleva diez años, por ahí es un paciente quemado, consulta a la guardia, se me metió una piedra en el camión y sentí un latigazo en el cuello y activás la emergencia... y no tenés nada, flaco. Pero modifican los hechos, buscan un descanso, un reposo durante la jornada, excusas. Empiezan a inventar patologías... no soportan más estar arriba. Y se quieren ir con la plata, entonces buscan el despido sin causa (profesional sanitarista, comunicación personal, 5 de febrero de 2019).

Por el contrario, están los que prefieren ocultar los síntomas, mentir y disimular el cansancio o el dolor para conservar los premios en el salario y el empleo.

En un estudio cuantitativo en el que se aplicaron 114 encuestas $^{2}$ en un proyecto en producción, realizado en 2019 por un equipo de investigación conformado por las autoras de este trabajo, se observaron dos fenómenos asociados al argumento desarrollado. En primer término, que la rotación ${ }^{3}$ en el empleo es una característica frecuente en la actividad minera metalífera, debido a la lejanía en las que se encuentran los proyectos mineros y al régimen de trabajo. Ello da como resultado que el $54 \%$ de los trabajadores registran una antigüedad menor a tres años en el proyecto, y dentro de ese grupo

\footnotetext{
1 Se refiere a catorce días de descanso por catorce días de trabajo.

2 Esta cifra corresponde a un $17 \%$ de los trabajadores de la Mina Gualcamayo, ubicada en la provincia de San Juan.

3 Tiempo en que los empleados permanecen en una empresa y frecuencia de reemplazo.
} 
un 35\% llevaba en ese momento menos de un año en la relación laboral. En segundo término, el $60,9 \%$ de los trabajadores cambiaría su trabajo por otro de igual o mejor salario que le permitiera estar más cerca de su familia.

Asimismo, en vinculación con las trayectorias laborales, en el informe final se afirma:

Frente a la perspectiva de continuar en la actividad minera en diez años más, en la amplia mayoría de los casos analizados los/las trabajadores/as mineros/ as señalaron sus deseos de no continuar en la actividad minera, incluso si percibieran un salario superlativo. $Y$ en sus argumentaciones, manifiestan el deseo de compartir la vida con la familia, los/las hijos/as que van creciendo y a quienes les sustentan estudios con su trabajo en la mina. La problemática relación entre la actividad minera y la trayectoria familiar llega también a expresarse como una incompatibilidad (Moscheni et al., 2019, p. 73).

Eso es así básicamente porque el trabajo y la familia son las dos instituciones más importantes que estructuran la vida de cualquier empleado que quiere conservar ambos, y en lo posible de manera armónica.

Pasados los catorce días de trabajo, los trabajadores se suben a un transporte que los llevará a sus hogares, cumpliendo con los catorce días de descanso previstos en el roster. Allí, el reencuentro con sus familias y demás vínculos afectivos es lo más importante, ya que pasaron la mitad del mes en completa ausencia física de ese entorno. Para los más jóvenes el primer fin de semana es tan intenso como su ritmo activo de trabajo. Uno de los entrevistados hace una narrativa simpática de esta trayectoria refiriéndose a su hijo "él baja un jueves, esa noche hasta el sábado o el domingo se lo llevan los marcianos y no sé dónde está, ya el domingo almuerza con nosotros, y hace cosas que debería hacer, lleva el auto al taller, por ejemplo" (empleado de Lama, comunicación personal, 16 de enero de 2019).

La actividad minera implicó una organización del trabajo completamente desconocida en San Juan hasta 2003, un pueblo pequeño del interior de la Argentina, cuyas actividades económicas más importantes, vinculadas esencialmente a la agroindustria, y el nivel de consumo de sus habitantes no eran atractivos hasta el momento para las grandes corporaciones trasnacionales. Los trabajadores sanjuaninos estaban acostumbrados a un ritmo de trabajo de ocho horas para los que tenían la suerte de estar en blanco y diez horas los informales, en semanas laborales que abarcaban desde el lunes hasta el sábado en la mañana y en las que se respetaban los feriados o fines de semana puente. La organización familiar es la típica de un lugar pequeño y conservador, familia biparental binaria argentina, la mujer cuida los hijos y el hombre de la casa es el encargado de tomar las decisiones importantes, además de hacer los asados los domingos, día de reunión festiva para la familia nuclear. 
La minería, una actividad mayoritariamente masculinizada, con sus lógicas trasnacionales de trabajo, vino a modificar esa dinámica: ahora habría quince días al mes en que el jefe de hogar ya no estaría para resolver las cuestiones vitales de la familia, se perdería el cumpleaños de un hijo, el primer día de la escuela, la caída de un diente, la Navidad e incluso tampoco estaría en los desenlaces más duros. Las trabajadoras mineras mujeres con hijos pequeños, signadas por una división de tareas patriarcal, atraviesan esta experiencia con más angustia que los hombres. No obstante, para todos, sin distinción de género, la lejanía del grupo afectivo es lo que se hace más difícil de llevar. A pesar de ello, la familia asume las consecuencias, comprende la nueva dinámica y se reorganiza, ya que a fin de mes el nuevo salario, más alto que el de cualquier otro trabajo posible en San Juan para un obrero, permite acceder a mayores y mejores consumos e incluso a una obra social que, hasta hace unos años atrás, solo era posible para ejecutivos. La mujer queda al cuidado exclusivo de los hijos, alternando el trabajo productivo y reproductivo. Luego, vendrán quince días en los que el trabajador minero estará a pleno nuevamente en el hogar, todos los días podrían ser domingos, pero ya nada será igual, en tanto si bien la presencia física se esperará con alegría, muchos episodios ocurridos durante la ausencia se convertirán en silencios y olvidos que con el tiempo serán potenciales vacíos familiares o episodios de violencia (los rumores de infidelidad en mineros que pasan mucho tiempo fuera de casa son comunes).

Así lo relata un trabajador jerárquico en edad de jubilarse:

Uno vuelve con tantas ganas, que si ve algo que está mal no abre la boca para no pelear, si total ya me voy... A su vez los chicos se portan de una manera cuando uno está y de otra cuando no está, suceden cosas que te las cuentan o no, y pasan los meses y cuentan algo y dicen "¿Te acordás?". Pero... "¿Y eso cuándo pasó?". Esas cosas se vuelven consuetudinarias, si uno no encuentra la ecuación para resolverlas, eso se termina convirtiendo en abismo, grietas de pareja o entre el hombre y sus hijos (empleado minero, comunicación personal, 16 de enero de 2019).

La nueva dinámica es la de una "familia acordeón", metáfora útil para ilustrar la situación de presencia activa del trabajador minero en su casa durante la mitad del mes, pero con una ausencia completa durante la otra mitad. Podría inferirse una categoría más de riesgo a las descritas por Gollac (2013), mencionadas en el apartado teórico de este artículo, los riesgos "sociofamiliares", cuyos síntomas se evidencian en silencios, culpas y angustia, y cuya consecuencia final puede desembocar en la ruptura de los vínculos familiares y sociales.

En este contexto, los trabajadores mineros adoptan una nueva familia, sus compañeros de trabajo, con quienes pasan gran parte de sus días. El tra- 
bajo en equipo, eslogan de muchas corporaciones en las que la capacitación en liderazgo es obligatoria incluso para un chofer de camión, permite cierta autonomía, concretamente en lo referido a la participación en la toma de decisiones de forma grupal. Las empresas abren más espacios de consulta entre los empleados, en áreas vinculadas al ahorro de costos, optimización de recursos y propuestas sobre una mejor forma de hacer las cosas. De esta manera, al consultar sobre la toma de decisiones, la mayoría de los entrevistados manifestaron hacerlas en equipo, combinando los conocimientos técnicos de algunos con el "saber hacer" no tácito de otros e intentando resolver los problemas cotidianos con creatividad. Esto revela cierto grado de autonomía, aun cuando todos coincidieron en respetar siempre la cadena de mando y los procedimientos o protocolos de acción. "En general en los/as empleados/as mineros/as directos/as se observa mayor autonomía y toma de decisiones en equipo de tipo calificante, originado en la intención de cooperación y superación" (Moscheni, 2021, p. 10).

Para finalizar, es preciso señalar que el flagelo del despido en la minería es constante, ya que se trata de una actividad que depende de variables externas, tales como el precio del oro y del dólar. Asimismo, al no ser un recurso renovable, se está frente a la constante posibilidad de que la extracción se acabe y, por tanto, que el proyecto termine. Por ello, los empleados tienen un contrato por tiempo "indeterminado", es decir, sin fecha precisa de finalización. El trabajo minero no es un trabajo precario, toda persona que ingrese a una mina, sea contratada directa o indirectamente, debe tener sus papeles en regla, pero sí carece de estabilidad, como producto de los factores mencionados (agotamiento del mineral o precio del oro) o por reestructuraciones del capital. Cuando un nuevo grupo adquiere una empresa, predomina entre los empleados cierta sensación de incertidumbre respecto a su futuro laboral, generando síntomas propios de la ansiedad. Esta situación se agrava en trabajadores no jerárquicos, no sindicalizados y mujeres, ya que conforman el grupo más prescindible y, por tanto, más vulnerable.

\section{Conclusiones}

Este artículo tiene como propósito identificar los riesgos psicosociales en la minería metalífera, desde una mirada interpretativa y particularmente cualitativa, tomando para ello la clasificación de Gollac (2011, 2013, citado en Neffa, p. 2017, p. 80).

Respecto a los riesgos generados como consecuencia de la cantidad, ritmo e intensidad del trabajo, en el apartado de análisis se aportaron datos empíricos, en primer término, sobre el esfuerzo realizado por los trabajadores, evidenciado en la cantidad de horas dedicadas y la configuración de los turnos, que provoca fundamentalmente estrés, ansiedad y perturbaciones en el sueño. 
En segundo lugar, en lo que se refiere a las exigencias emocionales no se han identificado significaciones de sentido que reflejan consecuencias psíquicas provenientes de insultos, acosos, amenazas o demás actos violentos.

En tercer término, en lo que respecta a la autonomía del trabajo, los entrevistados han referido a distintas situaciones laborales que reflejan cierto margen de autonomía y posibilidad de participación en la toma de decisiones. Con relación a ello, los trabajadores contratados de manera directa reconocen mayores posibilidades de promoción y recompensas monetarias, particularmente en lo que respecta a los plus.

En cuarto término, en relación con la inseguridad, los conflictos éticos solo aparecen relacionados con uno de los principales cuestionamientos a la actividad minera metalífera: si se contamina el medio ambiente o no. En general los trabajadores defienden la actividad que les ofrece un puesto de trabajo seguro frente a la mirada de los grupos sociales en la que están insertos (familia, amigos, vecinos, etcétera).

Asimismo, uno de los riesgos que más aparece es el referido a la inestabilidad laboral, en tanto la continuidad de la actividad $-\mathrm{y}$, por tanto, de los puestos de trabajo - depende de factores que no son controlables por los trabajadores, como por ejemplo el precio del oro y del dólar a nivel mundial o la existencia de minerales.

Consideramos que el riesgo que tuvo mayor frecuencia en los discursos de los trabajadores, que emerge como una variable no mencionada por Gollac y es, por tanto, el aporte más importante de este trabajo es el de la pérdida del vínculo familiar. En esta dimensión se destacan dos emergentes: una derivada del trabajo de campo, para calificar a las familias mineras como "acordeón", y otra del analítico, que aporta los "riesgos sociofamiliares". Estos pueden ir desde la alteración de la dinámica y organización familiar tradicional, pasando por la ansiedad y el estrés que implica estar separados de sus afectos la mitad de sus vidas, hasta la ruptura y separación, para los que están casados. El trabajador vuelve cansado a su hogar, sufre la ausencia de los eventos más importantes en la trayectoria de vida de las personas que ama, viviendo con culpa por ello y sintiendo en algunas oportunidades la exclusión de las vivencias sociofamiliares, hasta llegar al punto de experimentar incompatibilidad de sostener trabajo y familia a la vez, lo que deriva en el término de la relación laboral o en la separación matrimonial.

Por otro lado, la actividad registra un alto nivel de rotación, lo que incide en la antigüedad. Algunos trabajadores mineros siguen en la actividad y, aun a riesgo de perder la antigüedad acumulada, cambian de proyecto o empresa. Es frecuente que las operadoras desarrollen políticas de atracción de profesionales con trayectoria, experiencia y alta formación, en particular en puestos jerárquicos o mano de obra altamente calificada. Otros salen de la actividad, en algunos casos debido a variables externas a la decisión personal del asalariado, como la finalización de un proyecto o la caída de variables globales, como el precio in- 
ternacional del oro, que inciden de manera directa en las inversiones e indirecta en el empleo demandado. Aunque en menor proporción, se identificaron casos que priorizan los vínculos afectivos y ponen en marcha proyectos, en general comerciales, con el dinero ahorrado en la minería.

Por otra parte, en el plano institucional consideramos que los daños psicológicos y sociales que derivan de los distintos RPST tienen una atención limitada respecto a los físicos, por las empresas, los sindicatos y la normativa misma. El Decreto de Higiene y Seguridad sancionado específicamente para la actividad minera no los contempla. Otro ejemplo con relación a la normativa es el de la jubilación anticipada prevista para trabajos de alto riesgo, como aspecto clave para reducir el síntoma de "paciente quemado", presente en un trabajador que pasa la mitad de su vida haciendo trabajo de altura y lejos de su familia. Si bien las empresas podrían adherir al Decreto nacional 2338/69, que prevé un régimen de jubilación preferencial, esto es opcional. En la provincia de San Juan no hay una ley provincial que lo regule y si bien algunas empresas mineras no metalíferas se han adherido, las operadoras metalíferas no lo han hecho.

Los riesgos psicosociales y sus causas tampoco están previstos en las capacitaciones realizadas por los principales gremios en la mina, como AOMA, ASIJEMÍN, UOCRA o ASIMRA. De la misma manera, ninguno de los nueve conflictos gremiales que se realizaron en dieciséis años de producción (20032019) tuvo alguna reivindicación relacionada con este tema. Los trabajadores están expuestos a controles psicosociales al momento de ingresar a la mina, pero no existe una revisión sistemática posterior que permita un seguimiento.

No obstante, entendemos que el planteo de fondo es otro. Si bien las empresas cumplen algunas normativas, como las de contar con un servicio de prevención de riesgos, capacitación constante o entrega de elementos de protección personal, lo cierto es que todas esas prácticas están orientadas a la prevención o el resarcimiento de los riesgos físicos, pero no los psíquicos. Una explicación de ello se asienta sobre el predominio, en el modo de producción capitalista, de una política productivista, cuyo objetivo es evitar el riesgo para no interrumpir la producción. En efecto, los accidentes de trabajo dan cuenta de esa mirada al medirse por "tiempo perdido". Ello tiene como consecuencia que no existan prácticas codificadas en el ámbito laboral cotidiano ni establecidas por ley que prevean acciones para avanzar en la humanización del trabajo. Esto podría ser posible si los distintos actores reconocieran los mismos riesgos, pero, por el contrario, hay consecuencias que son consideradas riesgos por algunos y omitidas por otros.

Por último, es necesario desnaturalizar prácticas y discursos comunes, tales como que los seres humanos son una máquina y la salud una mercancía. Para ello, los sindicatos y el Estado tienen el desafío de identificar y detectar a tiempo los RPST, poner en agenda el tema, promover regulaciones que reemplacen los vacíos en las normas vigentes y capacitar e informar a los traba- 
jadores, atendiendo a uno de los desafíos contemporáneos más importantes, como lo es la humanización del trabajo, en particular en países en desarrollo como la Argentina.

\section{Referencias bibliográficas}

AOMA-BARRICK (2010). Convenio colectivo de trabajo (Veladero). San Juan: AOMA-BARRICK.

AOMA-Troy Resources Argentina LTD (2010). Convenio colectivo de trabajo. San Juan: AOMA-Troy Resources Argentina LTD. Disponible en: $<\mathrm{http} / / /$ enernews.com/nota/53721/documento-que-dice-el-convenio-laboral-aomatroy> [acceso 16/12/2020].

Bonino, D. (1979). El compañero médico. México: Nueva Imagen.

Basaglia, F. (1978). La salud de los trabajadores. México: Nueva Imagen.

De Souza Minayo, María Cecilia (2010). Los conceptos estructurantes de la investigación cualitativa. Salud Colectiva, 6(3), 251-261.

Fischer, F. M. (2018). Lidando com as longas jornadas de trabalho em turnos na mineração: desafios e possíveis intervenções. Revista Brasileira de Medicina Do Trabalho, 16(1), pp. 29-31.

Gollac, M. (2013). Los riesgos psicosociales en el trabajo. Exposición en el Seminario Internacional Los Riesgos Psico Sociales en el Trabajo. Facultad de Ciencias Económicas de la Universidad Nacional de La Plata, CEIL, Ministerio de Trabajo de la Provincia de Buenos Aires, La Plata.

Henry, M. L. (2019). Salud laboral en el escenario productivo actual: la creciente incidencia de los riesgos psicosociales. Revista de Ciencias Sociales, 32(44), pp. 171-196.

Knight Piésold Consulting (2002). Informe de impacto ambiental Veladero [en línea]. s. 1.: Knight Piésold Consulting. Disponible en: <http://center-hre. org/wp-content/uploads/2013/05/veladero-informe-de-impacto-ambientalexploracion.pdf> [acceso 24/05/2021].

Korinfeld, S. y N. Mendizábal (2015). Estudios sobre condiciones de trabajo, medio ambiente y su relación con la salud: los aportes del CEIL-CONICET. Voces en el Fénix, 46, pp. 90-97.

Laurell, A. C. (coord.) (1993). Para la investigación sobre la salud de los trabajadores. Washington, DC: Organización Panamericana de la Salud. 
Minera Argentina Gold S.A. (2002). Informe de impacto ambiental Proyecto Veladero. s. 1.: Minera Argentina Gold S.A.

Moscheni, M. (2021). Minería metalífera: un territorio de aprendizajes. El caso de la provincia de San Juan, Argentina. Revista Población \& Sociedad, 28(1).

Moscheni, M.; V. Gili Diez; D. de la Torre; J. Neffa; A. Ureta; E. Funes; F. Esquivel; M. Pérez; M. Grimalt; A. Checarelli; L. de los Ríos; A. Heredia; V. Godoy; R. Ortiz y L. Doncel (2019). Entramado productivo minero en San Juan. Herramientas de intervención desde un abordaje territorial. Proyecto de Desarrollo Tecnológico Social. Universidad Nacional de San Juan, Argentina.

Neffa, J. C. (2017). El marco teórico de la investigación. En: J. C. Neffa y M. L. Henry. ¿Quién cuida a los que cuidan? Los riesgos psicosociales en el trabajo en los establecimientos privados de salud. La Plata: Instituto de Investigaciones Administrativas, Facultad de Ciencias Económicas, Universidad Nacional de La Plata, pp. 16-114.

Neffa, J. C. (2015). Los riesgos psicosociales en el trabajo: contribución a su estudio. Buenos Aires: Centro de Estudios e Investigaciones Laborales-CEILCONICET.

Neffa, J. C. y M. L. Henry (2017). ¿Quién cuida a los que cuidan? Los riesgos psicosociales en el trabajo en los establecimientos privados de salud. La Plata: Instituto de Investigaciones Administrativas, Facultad de Ciencias Económicas, Universidad Nacional de La Plata.

Neiman, G. y G. Quaranta (2013). Los estudios de caso en la investigación sociológica. En: I. Vasilachis de Gialdino (coord.). Estrategias de investigación cualitativa. Buenos Aires: Gedisa, pp. 213-237.

Observatorio de Empleo y Dinámica Empresarial (2016). Boletín trimestral de empleo registrado por provincia [en línea]. Buenos Aires: Ministerio de Trabajo, Empleo y Seguridad Social. Disponible en: < http://www.trabajo. gob.ar/estadisticas/oede/estadisticasregionales.asp > [acceso 24/05/2021].

\section{Contribución de autoría}

Este trabajo fue realizado en partes iguales por Margarita Moscheni y Valeria Gili Diez. 